\title{
Cognitive Behavioral Therapy versus Eye Movement Desensitization and Reprocessing in Patients with Post- traumatic Stress Disorder: Systematic Review and Meta-analysis of Randomized Clinical Trials
}

Ali M. Khan ${ }^{1}$, Sabrina Dar ${ }^{2}$, Rizwan Ahmed ${ }^{3}$, Ramya Bachu ${ }^{4}$, Mahwish Adnan ${ }^{5}$, Vijaya Padma Kotapati 6

1. Psychiatry Resident, University of Texas Rio Grande Valley, Harlingen, USA 2. Psychiatry, Saint Elizabeth's Medical Center, Boston, USA 3. Psychiatry, Liaquat College, Karachi, PAK 4. Psychiatry, Northwell Zucker Hillside Hospital, New York, USA 5. Center for Addiction and Mental Health, University of Toronto, Toronto, CAN 6. Psychiatry, Manhattan Psychiatric Center, New York, USA

$\square$ Corresponding author: Vijaya Padma Kotapati, padmakotapati89@gmail.com Disclosures can be found in Additional Information at the end of the article

\section{Abstract}

\section{Background}

Post-traumatic stress disorder (PTSD) is prevalent in children, adolescents and adults. It can occur alone or in comorbidity with other disorders. A broad range of psychotherapies such as cognitive behavioral therapy (CBT) and eye movement desensitization and reprocessing (EMDR) have been developed for the treatment of PTSD.

\section{Aim}

Through quantitative meta-analysis, we aimed to compare the efficacy of CBT and EMDR: (i) relieving the post-traumatic symptoms, and (ii) alleviating anxiety and depression, in patients with PTSD.

\section{Methods}

We systematically searched EMBASE, Medline and Cochrane central register of controlled trials (CENTRAL) for articles published between 1999 and December 2017. Randomized clinical trials (RCTs) that compare CBT and EMDR in PTSD patients were included for quantitative meta-

Received 08/27/2018 Review began 08/31/2018 Review ended 08/31/2018 Published 09/04/2018

\section{(c) Copyright 2018}

Khan et al. This is an open access article distributed under the terms of the Creative Commons Attribution License CC-BY 3.0., which permits unrestricted use, distribution, and reproduction in any medium, provided the original author and source are credited. analysis using RevMan Version 5.

\section{Results}

Fourteen studies out of 714 were finally eligible. Meta-analysis of 11 studies $(n=547)$ showed that EMDR is better than CBT in reducing post-traumatic symptoms [SDM $(95 \% \mathrm{CI})=-0.43(-$ $0.73--0.12), p=0.006]$. However, meta-analysis of four studies $(n=186)$ at threemonth follow-up revealed no statistically significant difference [SDM $(95 \% \mathrm{CI})=-0.21(-0.50-$ $0.08), \mathrm{p}=0.15]$. The EMDR was also better than CBT in reducing anxiety [SDM $(95 \% \mathrm{CI})=-0.71$ $(-1.21--0.21), p=0.005]$. Unfortunately, there was no difference between CBT and EMDR in reducing depression [SDM $(95 \% \mathrm{CI})=-0.21(-0.44-0.02), \mathrm{p}=0.08]$.

\section{How to cite this article}

Khan A M, Dar S, Ahmed R, et al. (September 04, 2018) Cognitive Behavioral Therapy versus Eye Movement Desensitization and Reprocessing in Patients with Post-traumatic Stress Disorder: Systematic Review and Meta-analysis of Randomized Clinical Trials. Cureus 10(9): e3250. DOI 10.7759/cureus.3250 


\section{Conclusion}

The results of this meta-analysis suggested that EMDR is better than CBT in reducing posttraumatic symptoms and anxiety. However, there was no difference reported in reducing depression. Large population randomized trials with longer follow-up are recommended to build conclusive evidence.

Categories: Psychiatry, Psychology, Other

Keywords: eye movement desensitization and reprocessing (emdr), post-traumatic stress disorder (ptsd), cognitive behavioral therapy (cbt)

\section{Introduction}

The definition of post-traumatic stress disorder (PTSD) has evolved over time and available criteria differ in many ways. According to the diagnostic and statistical manual of mental disorders (DSM-V), a traumatic event is defined as the exposure to actual or threatened death, serious injury or sexual violation and leading to re-experiencing, avoidance, negative cognitions and mood, and arousal [1]. But the ICD-10 Diagnostic Criteria for Research (ICD-10DCR) only nominates events that lead to the distress of individuals [2]. The main variance between those two criteria is, the number of symptoms and duration of persistence. In addition, the DSM-V criteria require symptom persistence for more than a month whereas ICD10-DCR necessitates symptom persistence for more than six months. The PTSD can occur due to natural or human events; natural incidents include natural calamity, accidents, assault, terrorist attacks, and war, while the human events include sexual assault, sudden death, kidnapping, serious illness and disaster [3].

Few studies have reported a comparison of occurrence of traumatic events among different countries. A comparison of 16 countries found Netherlands, Colombia and the USA had the highest rates of trauma exposure while the lowest rates of exposure were found in Italy, Romania and Spain [4]. However, not all people who exposed to trauma develop PTSD. Moreover, the risk of developing PTSD varies among different sections of the population. Population sections that are at a higher risk of developing PTSD are females, those lacking social support, economically marginalized, and the younger at time of exposure [5]. A survey of participants aged between 15 and 54 years in the United States found more than $60 \%$ of males and more than half of the females had experienced a traumatic event [6].

In the United States, the prevalence of PTSD has been estimated to be 8.3\% [7]. Differences in prevalence of PTSD have documented in intentional and non-intentional trauma. Exposure to an intentional traumatic event increased the prevalence of PTSD over time while exposure to non-intentional events led to a decline in PTSD prevalence over time. In about $60 \%$ of individuals, traumatic symptoms resolve over time without any intervention. In case those patients developed PTSD, therapeutic options include psychotherapies and pharmacotherapies. Available psychotherapies are prolonged exposure, cognitive behaviour therapy (CBT) and eye movement desensitization and reprocessing (EMDR), while available pharmacotherapies are selective serotonin reuptake inhibitors (SSRI) [8].

The effectiveness of CBT in treating PTSD has been documented in many studies $[9,10]$. In addition, the CBT has shown to be effective at reducing PTSD scores in PTSD associated with other conditions such as depression and anxiety. Similarly, the EMDR is one of the best remedies of PTSD as per World Health Organization (WHO) and there is a mounting number of studies reporting the effectiveness of EMDR in treating PTSD [11, 12]. EMDR has proved to be an effective treatment for reducing negative emotions and arousal. Additionally, the recent trials have established that EMDR has an effective role in reducing anxiety and depression in 
PTSD patients $[3,13]$.

Several recent meta-analyses have compared the efficacy of CBT and EMDR among children, adolescents and adults. A meta-analysis of 11 trials found that EMDR was slightly better than CBT in decreasing post-traumatic symptoms [14]. While another meta-analysis reported that EMDR and CBT were efficacious in reducing post-traumatic symptoms and depression but the reduction in depression was not statistically significant [15]. In contrast, the study by Seidler and Wagner did not find either EMDR or CBT to be superior at reducing post-traumatic symptoms [11]. Due to the contradictory results, we aimed to conduct an updated systematic review and meta-analysis to compare the efficacy of EMDR versus PTSD in reducing posttraumatic symptoms, anxiety, and depression.

\section{Materials And Methods}

\section{Methods}

This systematic review and meta-analysis is carried out in accordance with guidelines issued by Preferred Reporting for Systematic Reviews and Meta-analyses (PRISMA).

\section{Literature search}

Our systematic searching was limited to articles published between 1999 and December 2017. The process of identifying relevant articles began by searching for EMBASE, Medline and Cochrane Central Register of Controlled Trials. A manual search of the references list of included articles was conducted to identify any articles not retrieved by the database searches.

\section{Inclusion and exclusion criteria}

Only studies that met the following criteria were included in this meta-analysis: (1) study participants were children, adolescents or adults; (2) studies published in the English language; (3) studies that reported comparative results from randomized controlled trials of CBT and EMDR groups; (4) studies that reported a diagnosis of PTSD in accordance to DSM-V or DSMIV or DSM-III; (4) studies that reported adequate data for calculation of design effect. The exclusion criteria were: (1) studies reporting treatment methodologies focused on conditions other than PTSD, such as depression, bipolar disorder, behavioral problems, substance abuse, psychosis, suicidal ideation, and substance dependence; (2) studies reporting other types of psychotherapies such as psychodynamic therapy, hypnotherapy, self-help, and biofeedback.

\section{Search}

The key terms used in the searching process met the guidelines of Medical Subject Heading Terms. The key words were: post-traumatic stress disorder, "PTSD”, eye movement desensitization and reprocessing, "EMDR”, Cognitive Behavioral Therapy, “CBT”. The Boolean connector AND was used to form different combinations of the keyword, see Table 1. 


\section{Cureus}

\begin{tabular}{|c|c|c|c|}
\hline Key term & $\begin{array}{l}\text { Number of } \\
\text { articles in } \\
\text { MEDLINE }\end{array}$ & $\begin{array}{l}\text { Number of } \\
\text { articles in } \\
\text { EMBASE }\end{array}$ & $\begin{array}{l}\text { Number of articles } \\
\text { in Cochrane } \\
\text { CENTRAL }\end{array}$ \\
\hline $\begin{array}{l}\text { Post-traumatic stress disorder AND EMDR and } \\
\text { reprocessing AND CBT }\end{array}$ & 35 & 29 & 2 \\
\hline PTSD AND EMDR AND CBT & 36 & 46 & 8 \\
\hline $\begin{array}{l}\text { PTSD AND eye movement desensitisation and reprocessing } \\
\text { AND CBT }\end{array}$ & 35 & 20 & 3 \\
\hline $\begin{array}{l}\text { PTSD AND eye movement desensitisation and reprocessing } \\
\text { AND Cognitive Behavioral Therapy }\end{array}$ & 110 & 7 & 4 \\
\hline $\begin{array}{l}\text { Post-traumatic stress disorder AND eye movement } \\
\text { desensitisation and reprocessing AND Cognitive Behavioral } \\
\text { Therapy }\end{array}$ & 106 & 6 & 3 \\
\hline $\begin{array}{l}\text { Post-traumatic stress disorder AND eye movement } \\
\text { desensitisation and reprocessing AND CBT }\end{array}$ & 34 & 20 & 2 \\
\hline
\end{tabular}

\section{TABLE 1: Number of articles retrieved.}

EMDR: Eye movement desensitization and reprocessing; CBT: Cognitive behavioral therapy; PTSD: Post-traumatic stress disorder.

\section{Study selection}

All articles identified from all the databases were imported into one Endnote library where all duplicates were removed. The unique study titles and abstracts were screened through two independent reviewers, to check if they met inclusion criteria. Studies that met the inclusion criteria were fully reviewed by a third contributor "Ramya Bachu", to check if they reported the required data. Studies that did not report the required data were excluded.

\section{Data extraction}

Data extraction was carried out by two reviewers “Ali Khan” and "Padma Kotapati”. A third author "Ramya Bachu” compared the data to identify any inconsistencies. Any discrepancies were resolved through consultation among all authors, which ensured accurate data extraction process. For each included article, the means, standard deviations and p values of pretreatment, post-treatment and follow-up were extracted. Post-traumatic symptoms were measured using the PTSD reaction index (PTSD-RI), children's response to trauma index (CRTI), child report of post-traumatic symptoms (CROPS), clinician-administered PTSD scale child/adolescent version (CAPS-CA), clinician-administered PTSD scale (CAPS), and structured interview for PTSD (SI-PTSD). Anxiety was measured using hospital anxiety and depression scale (HADS), multidimensional anxiety scale for children (MASC), state-trait anxiety inventory (STAI), and revised children's anxiety and depression scale (RCADS-C). Depression was measured using the Beck depression index (BDI). Other extracted variables were: age, gender, CBT variants, treatment fidelity, comorbidity, co-interventions, study duration, year of publication, and methodological characteristics useful in assessing study bias. 


\section{Cureus}

\section{Results}

\section{Literature search}

The number of articles found in each database through using of various key terms is shown in Table 1. Of all screened papers, 14 articles were finally eligible for meta-analysis. The flow diagram of studies' selection is shown in Figure 1.

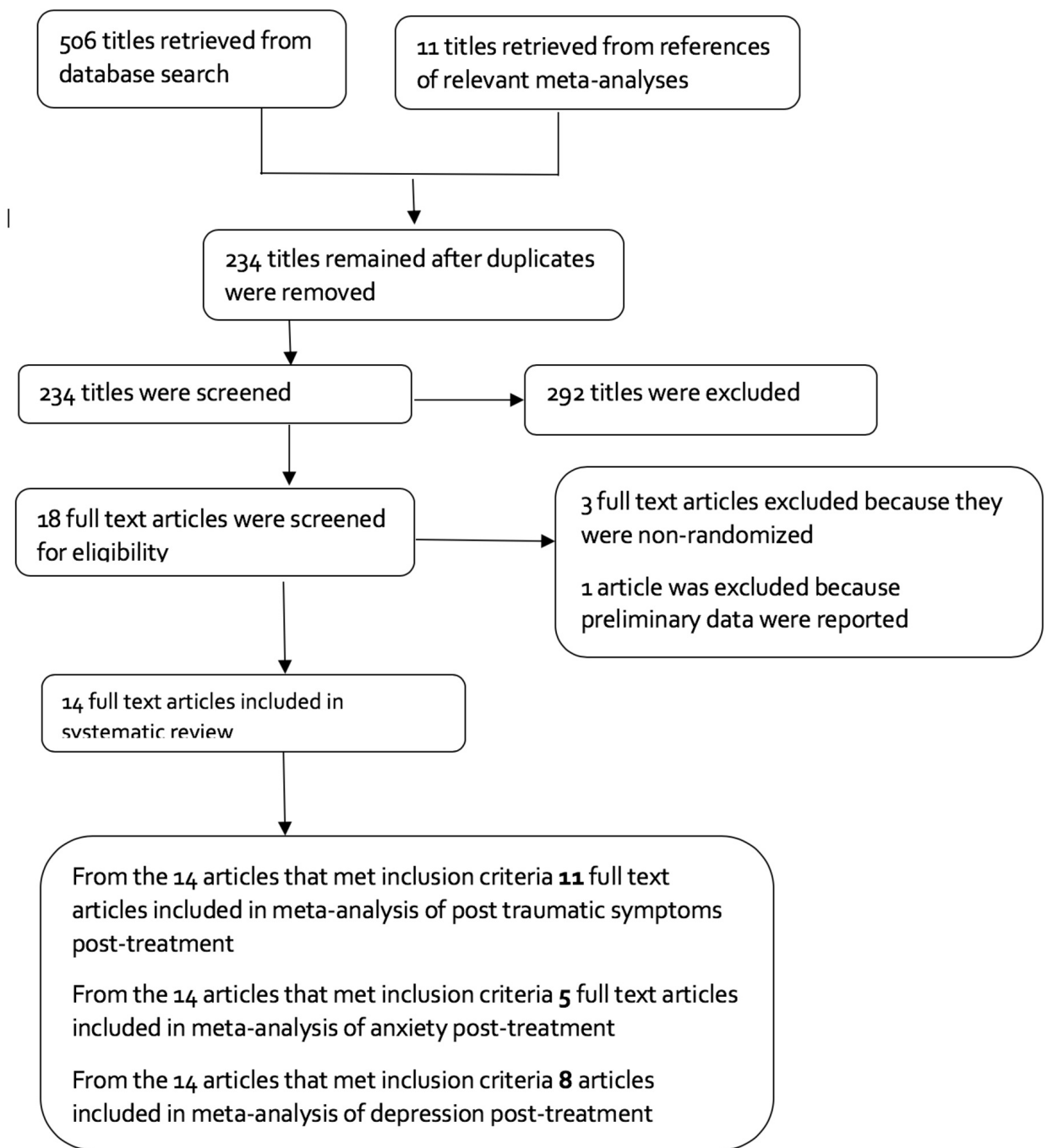

FIGURE 1: Selection of studies.

\section{Baseline characters}

The characteristics of included studies are shown in Table 2. The variants of CBT used were Imaginal Exposure (IE), Trauma Treatment Protocol (TTP), Prolonged exposure (PE), TraumaFocused Cognitive Behavioral Therapy (TF-CBT), Stress Inoculation Training with Prolonged Exposure (SITPE), Exposure plus cognitive restructuring $(\mathrm{E}+\mathrm{CR})$, and brief eclectic psychotherapy. Some patients had comorbidities in two studies by van den Berg (2015) and de Roos (2017). Nine studies used "intent to treat" to minimize the impact of participants who dropped out. Eight studies used treatment fidelity to ensure adherence to the treatment protocol. 


\section{Cureus}

\begin{tabular}{|c|c|c|c|c|c|c|c|c|c|c|}
\hline Study & Variants of CBT & $\begin{array}{l}\text { Trauma } \\
\text { type }\end{array}$ & $\begin{array}{l}\text { Clinician-rated } \\
\text { measures }\end{array}$ & Self-rated measures & $\begin{array}{l}\text { Mean } \\
\text { age } \\
\text { (years) }\end{array}$ & Males/Females & $\begin{array}{l}\text { Months } \\
\text { post }\end{array}$ & $\begin{array}{l}\text { Intention- } \\
\text { to-treat } \\
\text { (ITT) } \\
\text { analysis }\end{array}$ & $\begin{array}{l}\text { Treatment } \\
\text { fidelity } \\
\text { check }\end{array}$ & Comorbidity \\
\hline $\begin{array}{l}\text { Arabia et al., } \\
2011[13]\end{array}$ & Imaginal Exposure & $\mathrm{N} / \mathrm{A}$ & & IES-R, STAI, BDI & 63.48 & $28 / 14$ & 6 & Yes & Yes & Not reported \\
\hline $\begin{array}{l}\text { De Roos et } \\
\text { al., } 2011 \text { [16] }\end{array}$ & & & & $\begin{array}{l}\text { PTSD-RI, CROPS, PROPS, } \\
\text { BDS, MASC, CBCL }\end{array}$ & 10.1 & $29 / 23$ & 3 & Yes & No & Not reported \\
\hline $\begin{array}{l}\text { Devilly et al., } \\
1999[17]\end{array}$ & $\begin{array}{l}\text { Trauma Treatment } \\
\text { Protocol }\end{array}$ & Mixed & & $\begin{array}{l}\text { STAI-Y2, BDI, SCL-90=R, SUD, } \\
\text { PPD, CMS, IES, PSS-SR, CEQ, } \\
\text { DEVS-T }\end{array}$ & 37.96 & $8 / 15$ & 3 & No & Yes & Not reported \\
\hline $\begin{array}{l}\text { Ironson et al., } \\
2002[18]\end{array}$ & Prolonged Exposure & Mixed & & PSS-SR, BDI, DES, SUD & & $5 / 17$ & 3 & No & Yes & Not reported \\
\hline $\begin{array}{l}\text { Jaberghaderi } \\
\text { et al., } 2004 \\
\text { [19] }\end{array}$ & Trauma Focused-CBT & $\begin{array}{l}\text { Sexual } \\
\text { assault }\end{array}$ & $\begin{array}{l}\text { Rutter teacher } \\
\text { scale }\end{array}$ & CROPS, PROPS & 12.5 & 0/14 & & No & No & Not reported \\
\hline $\begin{array}{l}\text { Diehle et al., } \\
2015 \text { [3] }\end{array}$ & & Mixed & CAPS-CA & CRIES 13, RCADS, & 12.9 & $18 / 30$ & & Yes & Yes & Not reported \\
\hline $\begin{array}{l}\text { Lee et al., } \\
2002[20]\end{array}$ & $\begin{array}{l}\text { Stress Inoculation } \\
\text { Training and Prolonged } \\
\text { Exposure }\end{array}$ & Mixed & SI-PTSD, MMPI-K, & IES, BDI & 34.0 & 13/11 & 3 & No & Yes & Not reported \\
\hline $\begin{array}{l}\text { Power et al., } \\
2002[21]\end{array}$ & $\begin{array}{l}\text { Exposure + } \\
\text { Cognitive Restructuring }\end{array}$ & Mixed & $\begin{array}{l}\text { CAPS, MADRS, } \\
\text { HAM-A }\end{array}$ & $\begin{array}{l}\text { IOE, SI-PTSD, HADS, Sheehan } \\
\text { disability index }\end{array}$ & 40.9 & $42 / 30$ & 15 & No & No & Not reported \\
\hline $\begin{array}{l}\text { De Roos et } \\
\text { al., } 2017 \text { [22] }\end{array}$ & & & ADIS-C/P & $\begin{array}{l}\text { CRTI, C-PTCI, RCADS-C/P, } \\
\text { SDQ-A/P, CSI-C/P }\end{array}$ & 13.06 & $44 / 59$ & 12 & Yes & Yes & $\begin{array}{l}\text { Anxiety } \\
\text { disorders }\end{array}$ \\
\hline $\begin{array}{l}\text { Rothbaum et } \\
\text { al., } 2005 \text { [12] }\end{array}$ & Prolonged Exposure & $\begin{array}{l}\text { Sexual } \\
\text { assault }\end{array}$ & $\begin{array}{l}\text { CAPS, SLESQ, } \\
\text { SCID non-patient } \\
\text { version }\end{array}$ & $\begin{array}{l}\text { PSS-SR, IES-R, BDI, DES-II, } \\
\text { STAI }\end{array}$ & 33.8 & $0 / 60$ & 6 & Yes & No & Not reported \\
\hline $\begin{array}{l}\text { Taylor et al., } \\
2003 \text { [23] }\end{array}$ & Exposure & Mixed & CAPS & BDI & 37 & $15 / 45$ & 3 & Yes & No & Not reported \\
\hline $\begin{array}{l}\text { Nijdam et al., } \\
2012[24]\end{array}$ & $\begin{array}{l}\text { Brief eclectic } \\
\text { psychotherapy }\end{array}$ & $\mathrm{N} / \mathrm{A}$ & & IES-R, SI-PTSD, SCID-I, HADS & & & & Yes & Yes & Not reported \\
\hline $\begin{array}{l}\text { Capezzani et } \\
\text { al., } 2013 \text { [25] }\end{array}$ & & & CAPS & BDI, STAI-Y, QPF-R, IES-R & & & & No & No & Not reported \\
\hline $\begin{array}{l}\text { Van den Berg } \\
\text { et al., } 2015 \\
{[26]}\end{array}$ & Prolonged Exposure & Mixed & CAPS & PSS-SR, PTCI & 41.2 & $84 / 71$ & 6 & Yes & Yes & $\begin{array}{l}\text { Psychotic } \\
\text { disorder }\end{array}$ \\
\hline
\end{tabular}

\section{TABLE 2: Characteristics of included studies.}

N/A: Not applicable; PTSD: Post-traumatic stress disorder; CAPS: Clinician-administered PTSD Scale; PTSD-RI: PTSD-reaction index; 


\section{Cureus}

CRTI: Children's response to trauma index; CROPS: Child report of post-traumatic symptoms; PROPS: Parent report of post-traumatic stress symptoms; CAPS-CA: Clinician-administered PTSD scale child/adolescent version; SI-PTSD: Structured interview for PTSD; HADS: Hospital anxiety and depression scale; MASC: Multidimensional anxiety scale for children; STAl: State-trait anxiety inventory; RCADS-C: Revised children's anxiety and depression scale; BDI: Becks depression inventory; IES-R: Impact of event scale-revised; BDS: Backward digit span; CBCL: Child behavior checklist; STAI-Y2: State-Trait Anxiety Inventory-Y2 Trait Form; SUD: Substance use disorder; CMS: The Mississippi Scale for Civilian PTSD; PSS-SR: PTSD symptom scale self-report version; CEQ: Combat experiences questionnaire; DEVS-T: Distress evaluation scale for treatment; DES: Dissociative experiences scale; CRIES 13: Children's revised impact of event scale; RCADS: Revised child anxiety and depression scale; IOE: Impact of events scale; HADS: Hospital anxiety and depression score; CRTI: Children's responses to trauma inventory; C-PTCI: Children's post-traumatic cognitions inventory; RCADSC/P: Revised children's anxiety and depression scale- children/parent version; SDQ-A/P: Strengths and difficulties questionnaire; CSIC/P: Coping strategies inventory- Child/Parent; DES II: Dissociative experiences scale-II; QPF-R: The psychophysiological questionnaire-brief version; SCL-90 = R: Symptom checklist-90-Revised; SLESQ: Stressful life events screening questionnaire; SCID: Structured clinical interview for DSM-5; ADIS-C/P: Anxiety disorder interview schedule for DSM-IV: Child and parent interview schedule; MADRS: Montgomery Åsberg depression rating scale; MMPI-K: Minnesota multiphasic personality inventory-K scale; TFCBT: Trauma-focused cognitive behavioral therapy; HAM-A: Hamilton anxiety rating scale; CAPS-CA: Clinician-administered PTSD scale based upon DSM-5; PPD: Postpartum depression.

\section{Quality assessment}

All studies were assessed for risk of bias through the Cochrane tool. The risk of bias was defined as low, medium, high, and unclear. All studies reported random sequence allocation. There was a high risk of bias in four studies regarding blinding of the outcome. Only four studies reported allocation concealment. Detailed risk of bias assessment is shown in Table 3.

\begin{tabular}{|c|c|c|c|c|c|c|c|c|}
\hline \multirow{2}{*}{$\begin{array}{l}\text { Domain } \\
\text { Study }\end{array}$} & \multicolumn{2}{|c|}{ Allocation concealment } & \multicolumn{2}{|c|}{$\begin{array}{l}\text { Blinding of outcome } \\
\text { assessment }\end{array}$} & \multicolumn{2}{|c|}{ Incomplete outcome data } & \multicolumn{2}{|c|}{ Selective reporting } \\
\hline & Judgment & $\begin{array}{l}\text { Support for } \\
\text { judgment }\end{array}$ & Judgment & $\begin{array}{l}\text { Support for } \\
\text { judgment }\end{array}$ & Judgment & Support for judgment & Judgment & $\begin{array}{l}\text { Support } \\
\text { for } \\
\text { judgment }\end{array}$ \\
\hline $\begin{array}{l}\text { Arabia et al., } \\
2011 \text { [13] }\end{array}$ & Unclear & Not reported & Unclear & Not reported & Low risk & $\begin{array}{l}\text { Five participants in } \\
\text { EMDR and three } \\
\text { participants in IE lost } \\
\text { to follow up at month } \\
6 \text {. ITT was used. }\end{array}$ & Low risk & $\begin{array}{l}\text { All } \\
\text { measures } \\
\text { were } \\
\text { reported }\end{array}$ \\
\hline $\begin{array}{l}\text { De Roos et } \\
\text { al., } 2011 \text { [16] }\end{array}$ & Unclear & Not reported & Low risk & $\begin{array}{l}\text { Assessor was } \\
\text { blinded to } \\
\text { treatment } \\
\text { conditions }\end{array}$ & Low risk & $\begin{array}{l}\text { Eight participants in } \\
\text { EMDR group dropped } \\
\text { out and six participants } \\
\text { in CBT group dropped } \\
\text { out. ITT and } \\
\text { imputation used to } \\
\text { account for missing } \\
\text { observations }\end{array}$ & Low risk & $\begin{array}{l}\text { All } \\
\text { measures } \\
\text { were } \\
\text { reported }\end{array}$ \\
\hline $\begin{array}{l}\text { Devilly and } \\
\text { Spence, } \\
1999 \text { [17] }\end{array}$ & Unclear & Not reported & Unclear & Not reported & High risk & $\begin{array}{l}\text { Three participants in } \\
\text { TTP group dropped } \\
\text { out and six participants } \\
\text { in EMDR group } \\
\text { dropped out. Impact of } \\
\text { drop out was not } \\
\text { discussed }\end{array}$ & Low risk & $\begin{array}{l}\text { All } \\
\text { measures } \\
\text { were } \\
\text { reported }\end{array}$ \\
\hline
\end{tabular}




\section{Cureus}

\begin{tabular}{|c|c|c|c|c|c|c|c|c|}
\hline $\begin{array}{l}\text { Ironson et } \\
\text { al., } 2002 \text { [18] }\end{array}$ & Unclear & Not reported & High risk & $\begin{array}{l}\text { Assessors were } \\
\text { not blinded to } \\
\text { treatment } \\
\text { conditions }\end{array}$ & & $\begin{array}{l}\text { Three participants in } \\
\text { PE dropped out and } \\
\text { there was no drop out } \\
\text { in EMDR }\end{array}$ & Low risk & $\begin{array}{l}\text { All } \\
\text { measures } \\
\text { were } \\
\text { reported }\end{array}$ \\
\hline $\begin{array}{l}\text { Jaberghaderi } \\
\text { et al., } 2004 \\
\text { [19] }\end{array}$ & Unclear & Not reported & Low risk & $\begin{array}{l}\text { Screening was } \\
\text { done by } \\
\text { psychologists } \\
\text { blinded to } \\
\text { treatment }\end{array}$ & Low risk & $\begin{array}{l}\text { One participant in } \\
\text { EMDR and one } \\
\text { participant in CBT } \\
\text { dropped out. This was } \\
\text { a low drop out }\end{array}$ & Low risk & $\begin{array}{l}\text { All } \\
\text { measures } \\
\text { were } \\
\text { reported }\end{array}$ \\
\hline $\begin{array}{l}\text { Diehle et al., } \\
2015 \text { [3] }\end{array}$ & Low risk & $\begin{array}{l}\text { A researcher not } \\
\text { involved in study } \\
\text { managed } \\
\text { randomization list } \\
\text { and communicated } \\
\text { to therapist }\end{array}$ & Low risk & $\begin{array}{l}\text { Assessors were } \\
\text { blinded to } \\
\text { treatment }\end{array}$ & Low risk & $\begin{array}{l}12 \text { participants lost to } \\
\text { follow up. ITT and } \\
\text { imputation were used } \\
\text { to handle missing } \\
\text { observations }\end{array}$ & Low risk & $\begin{array}{l}\text { All } \\
\text { measures } \\
\text { were } \\
\text { reported }\end{array}$ \\
\hline $\begin{array}{l}\text { Lee et } \\
\text { al., } 2002 \text { [20] }\end{array}$ & Unclear & Not reported & High risk & $\begin{array}{l}\text { Assessor was } \\
\text { blinded at pre- } \\
\text { treatment but not } \\
\text { at post-treatment } \\
\text { and follow-up }\end{array}$ & Low risk & $\begin{array}{l}\text { One participant from } \\
\text { SITPE and one } \\
\text { participant from EMDR } \\
\text { dropped out. This was } \\
\text { a minimal drop out }\end{array}$ & Low risk & $\begin{array}{l}\text { All } \\
\text { measures } \\
\text { were } \\
\text { reported }\end{array}$ \\
\hline $\begin{array}{l}\text { Power et al., } \\
2002 \text { [21] }\end{array}$ & Low risk & $\begin{array}{l}\text { Sealed envelope } \\
\text { was used to } \\
\text { conceal } \\
\text { randomization } \\
\text { group }\end{array}$ & $\begin{array}{l}\text { Medium } \\
\text { risk }\end{array}$ & $\begin{array}{l}\text { Pre- and post- } \\
\text { treatment } \\
\text { assessors were } \\
\text { blinded but there } \\
\text { was no blind mid- } \\
\text { point and at } \\
\text { follow-up }\end{array}$ & $\begin{array}{l}\text { Medium } \\
\text { risk }\end{array}$ & $\begin{array}{l}12 \text { participants in } \\
\text { EMDR dropped out, } 16 \\
\text { in E+CR and five in } \\
\text { WL. Impact of dropped } \\
\text { participants not clear }\end{array}$ & Low risk & $\begin{array}{l}\text { All } \\
\text { measures } \\
\text { were } \\
\text { reported }\end{array}$ \\
\hline $\begin{array}{l}\text { De Roos et } \\
\text { al., } 2017 \text { [22] }\end{array}$ & Low risk & $\begin{array}{l}\text { Opaque sealed } \\
\text { envelopes } \\
\text { containing cards } \\
\text { with trial arms were } \\
\text { used to conceal } \\
\text { allocation }\end{array}$ & Low risk & $\begin{array}{l}\text { Independent } \\
\text { assessors were } \\
\text { blinded to } \\
\text { treatment }\end{array}$ & Unclear & Not reported & Low risk & $\begin{array}{l}\text { All } \\
\text { measures } \\
\text { were } \\
\text { reported }\end{array}$ \\
\hline $\begin{array}{l}\text { Rothbaum et } \\
\text { al., } 2005 \text { [12] }\end{array}$ & Unclear & Not reported & Low risk & $\begin{array}{l}\text { Assessors were } \\
\text { blinded to } \\
\text { treatment }\end{array}$ & Low risk & $\begin{array}{l}12 \text { participants } \\
\text { dropped out of the } \\
\text { study. PE = 3, EMDR } \\
=5, \text { WAIT }=4 . \text { ITT did } \\
\text { not provide different } \\
\text { results. }\end{array}$ & Low risk & $\begin{array}{l}\text { All } \\
\text { measures } \\
\text { were } \\
\text { reported }\end{array}$ \\
\hline $\begin{array}{l}\text { Taylor et al., } \\
2003 \text { [23] }\end{array}$ & Unclear & Not reported & Low risk & $\begin{array}{l}\text { Interviewers were } \\
\text { blinded to } \\
\text { treatment }\end{array}$ & & $\begin{array}{l}\text { Five patients in EMDR } \\
\text { and seven patients in } \\
\text { PE dropped out. }\end{array}$ & Unclear & $\begin{array}{l}\text { Not } \\
\text { reported }\end{array}$ \\
\hline $\begin{array}{l}\text { Nijdam et al., } \\
2012 \text { [24] }\end{array}$ & Low risk & $\begin{array}{l}\text { A psychologist not } \\
\text { involved in study } \\
\text { had randomization }\end{array}$ & Low risk & $\begin{array}{l}\text { Assessors were } \\
\text { masked to }\end{array}$ & High risk & $\begin{array}{l}20 \text { participants in } \\
\text { EMDR and } 25 \\
\text { participants in brief } \\
\text { eclectic therapy }\end{array}$ & & \\
\hline
\end{tabular}




\section{Cureus}

\begin{tabular}{|c|c|c|c|c|c|c|c|c|}
\hline & & file & & treatment group & & $\begin{array}{l}\text { dropped out. Although } \\
\text { ITT was used this was } \\
\text { a significant drop out }\end{array}$ & & \\
\hline $\begin{array}{l}\text { Capezzani et } \\
\text { al., } 2013 \text { [25] }\end{array}$ & Unclear & Not reported & Low risk & $\begin{array}{l}\text { CAPS was } \\
\text { administered by } \\
\text { blind assessor }\end{array}$ & Low risk & No patient dropouts & Low risk & $\begin{array}{l}\text { All } \\
\text { measures } \\
\text { were } \\
\text { reported }\end{array}$ \\
\hline $\begin{array}{l}\text { Van den } \\
\text { Berg et al., } \\
2015 \text { [26] }\end{array}$ & Unclear & Not reported & . & $\begin{array}{l}\text { There was } \\
\text { blinding but } 27 \\
\text { incidences of un- } \\
\text { blinding occurred } \\
\text { and these } \\
\text { assessments were } \\
\text { repeated }\end{array}$ & Low risk & $\begin{array}{l}13 \text { participants in PE } \\
\text { and } 11 \text { participants in } \\
\text { EMDR dropped out. } \\
\text { Completer analyses } \\
\text { and ITT did not yield } \\
\text { different results }\end{array}$ & Unclear & $\begin{array}{l}\text { BDI } \\
\text { scores } \\
\text { not } \\
\text { reported } \\
\text { post- } \\
\text { treatment } \\
\text { and at six } \\
\text { month } \\
\text { follow-up }\end{array}$ \\
\hline
\end{tabular}

\section{TABLE 3: Detailed risk of bias assessment.}

EMDR: Eye movement desensitization and reprocessing; IE: Imaginal exposure; ITT: Intention to treat; CBT: Cognitive behavioral therapy; TTP: Trauma treatment protocol; PE: Prolonged exposure; SITPE: Stress inoculation training with prolonged exposure; E+ CR: Exposure plus cognitive restructuring; WL: Waiting list; WAIT: No treatment wait list control.

\section{Meta-analysis of post-traumatic symptoms post-treatment}

Pooling 11 studies in a meta-analysis, the EMDR was better than CBT in reducing posttraumatic symptoms [SDM $(95 \% \mathrm{CI})=-0.43(-0.73--0.12)]$. The results were statistically significant ( $\mathrm{p}=0.006$ ); however, the studies included in this quantitative meta-analysis had a high level of heterogeneity (I2 $=62 \%$ ) mentioned in Figure 2 . Also, a funnel plot of publication bias did not show any asymmetry and no bias among included studies mentioned in Figure 3.

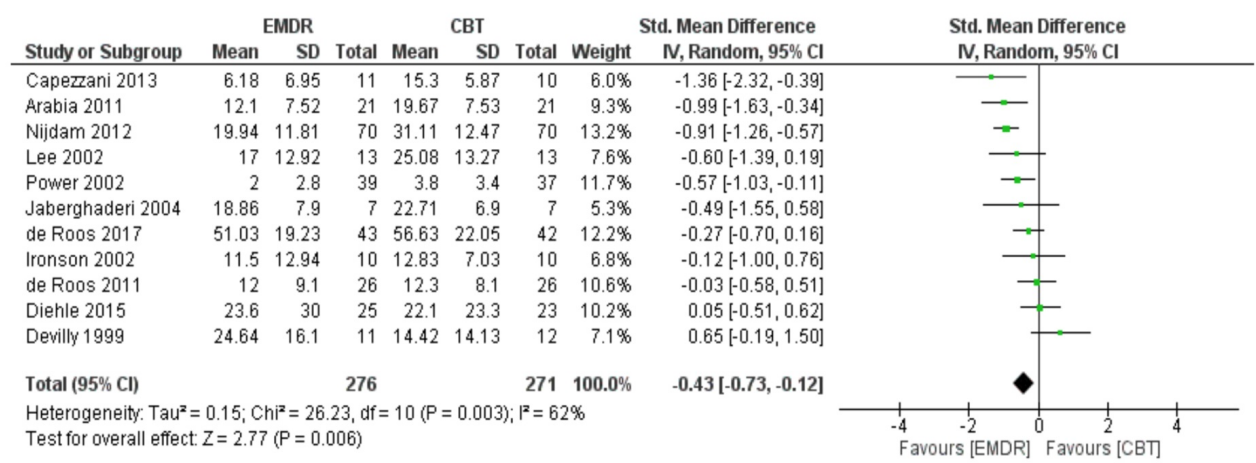

FIGURE 2: Meta-analysis of post-traumatic symptoms posttreatment.

EMDR: Eye movement desensitization and reprocessing; CBT: Cognitive behavioral therapy. 


\section{Cureus}

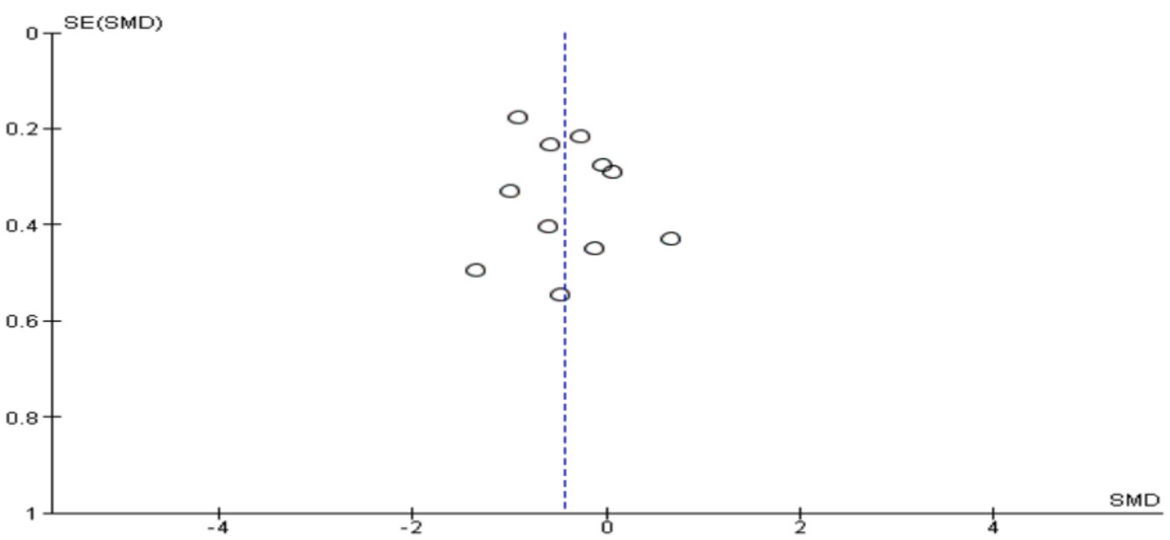

FIGURE 3: Funnel plot for meta-analysis of post-traumatic symptoms post-treatment.

\section{Meta-analysis of PTSD symptoms at three months follow-up}

At three months follow-up, the meta-analysis of four studies showed that EMDR was not better than CBT in reducing post-traumatic symptoms [SDM $(95 \% \mathrm{CI})=-0.21(-0.50-0.08), \mathrm{p}=0.15$ ] mentioned in Figure 4. Also, a funnel plot of publication bias of this meta-analysis did not show any asymmetry and no bias among included studies in Figure 5.

\begin{tabular}{|c|c|c|c|c|c|c|c|c|c|}
\hline \multirow[b]{2}{*}{ Study or Subgroup } & \multicolumn{3}{|c|}{ EMDR } & \multicolumn{2}{|r|}{ CBT } & \multicolumn{3}{|c|}{ Std. Mean Difference } & \multirow{2}{*}{$\begin{array}{c}\text { Std. Mean Difference } \\
\text { N, Fixed, } 95 \% \mathrm{Cl}\end{array}$} \\
\hline & Mean & SD & Total & Mean & SD & Total & Weight & N, Fixed, $95 \%$ Cl & \\
\hline de Roos 2011 & 11.2 & 8 & 26 & 11.9 & 8.3 & 26 & $28.4 \%$ & $-0.08[-0.63,0.46]$ & \\
\hline de Roos 2017 & 51.96 & 20.58 & 51 & 54.3 & 20.22 & 45 & $52.2 \%$ & $-0.11[-0.51,0.29]$ & \\
\hline Ironson 2002 & 11.5 & 12.94 & 6 & 15.67 & 4.93 & 6 & $6.4 \%$ & $-0.39[-1.54,0.75]$ & \\
\hline Lee 2002 & 14.17 & 12.15 & 13 & 24.33 & 12.03 & 13 & $13.0 \%$ & $-0.81[-1.62,-0.01]$ & \\
\hline Total (95\% Cl) & & & 96 & & & 90 & $100.0 \%$ & $-0.21[-0.50,0.08]$ & \\
\hline \multicolumn{9}{|c|}{$\begin{array}{l}\text { Heterogeneity: } C \mathrm{Ch}^{2}=2.68, \mathrm{df}=3(P=0.44) ; 1^{2}=0 \% \\
\text { Test for overall effect: } Z=1.45(P=0.15)\end{array}$} & $\begin{array}{cccr}-1 & 0 & 1 & 2 \\
\text { urs [EMDRI] } & \text { Favours [CBT] }\end{array}$ \\
\hline
\end{tabular}

\section{FIGURE 4: Meta-analysis of PTSD symptoms at three months} follow-up.

EMDR: Eye movement desensitization and reprocessing; CBT: Cognitive behavioral therapy; PTSD: Post-traumatic stress disorder. 


\section{Cureus}

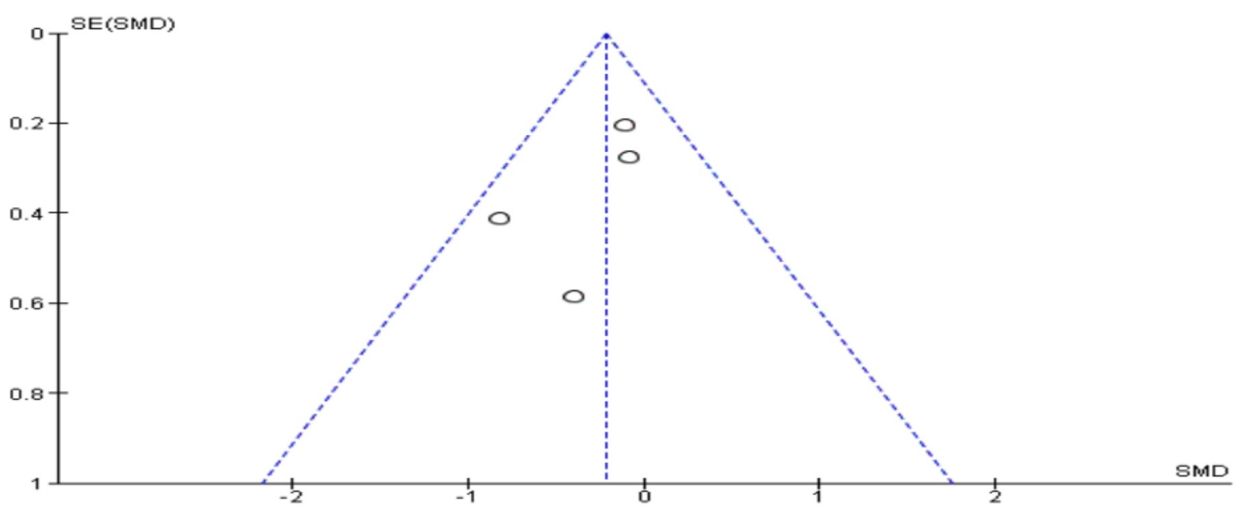

FIGURE 5: Funnel plot of meta-analysis of PTSD symptoms at three months follow-up.

PTSD: Post-traumatic stress disorder.

\section{Meta-analysis of anxiety post-treatment}

The meta-analysis of five studies including 239 patients revealed that EMDR is better than CBT in reducing anxiety symptoms [SDM $(95 \% \mathrm{CI})=-0.71(-1.21--0.21)]$. The result was statistically significant $(\mathrm{p}=0.005)$ but there was marked heterogeneity between included studies (I2 = 70\%) in Figure 6. Of note, there was no evidence of publication bias in the included studies in Figure 7.

\begin{tabular}{|c|c|c|c|c|c|c|c|c|c|}
\hline \multirow[b]{2}{*}{ Study or Subgroup } & \multicolumn{3}{|c|}{ EMDR } & \multicolumn{2}{|r|}{ CBT } & \multicolumn{3}{|r|}{ Std. Mean Difference } & \multirow{2}{*}{$\begin{array}{c}\text { Std. Mean Difference } \\
\text { N, Random, } 95 \% \mathrm{Cl}\end{array}$} \\
\hline & Mean & SD & Total & Mean & SD & Total & Weight & N, Random, 95\% Cl & \\
\hline Diehle 2015 & 1.6 & 1.3 & 25 & 5.6 & 3.5 & 23 & $19.7 \%$ & $-1.52[-2.16,-0.87]$ & $\rightarrow$ \\
\hline Arabia 2011 & 33.1 & 7.75 & 21 & 40.19 & 7.77 & 21 & $19.9 \%$ & $-0.90[-1.53,-0.26]$ & $\rightarrow$ \\
\hline Capezzani 2013 & 40 & 3.41 & 11 & 43.9 & 5.55 & 10 & $15.0 \%$ & $-0.82[-1.72,0.08]$ & \\
\hline Power 2002 & 7.7 & 5.1 & 39 & 10.3 & 6.8 & 37 & $23.6 \%$ & $-0.43[-0.89,0.03]$ & -4 \\
\hline de Roos 2011 & 33.1 & 14.9 & 26 & 33.8 & 18.9 & 26 & $21.8 \%$ & $-0.04[-0.58,0.50]$ & \\
\hline Total (95\% Cl) & & & 122 & & & 117 & $100.0 \%$ & $-0.71[-1.21,-0.21]$ & A \\
\hline \multicolumn{9}{|c|}{$\begin{array}{l}\text { Heterogeneity. } \text { Tau }^{2}=0.22 ; \mathrm{Ch}^{2}=13.26, \mathrm{df}=4(P=0.01) ; \mathrm{I}^{2}=70 \% \\
\text { Test for overall effect: } Z=2.79(P=0.005)\end{array}$} & $\begin{array}{ccccc}-4 & -2 & 0 & 2 & 4 \\
\text { urs [EMDR] } & \text { Favours [CE }\end{array}$ \\
\hline
\end{tabular}

FIGURE 6: Meta-analysis of anxiety post-treatment.

EMDR: Eye movement desensitization and reprocessing; CBT: Cognitive behavioral therapy. 


\section{Cureus}

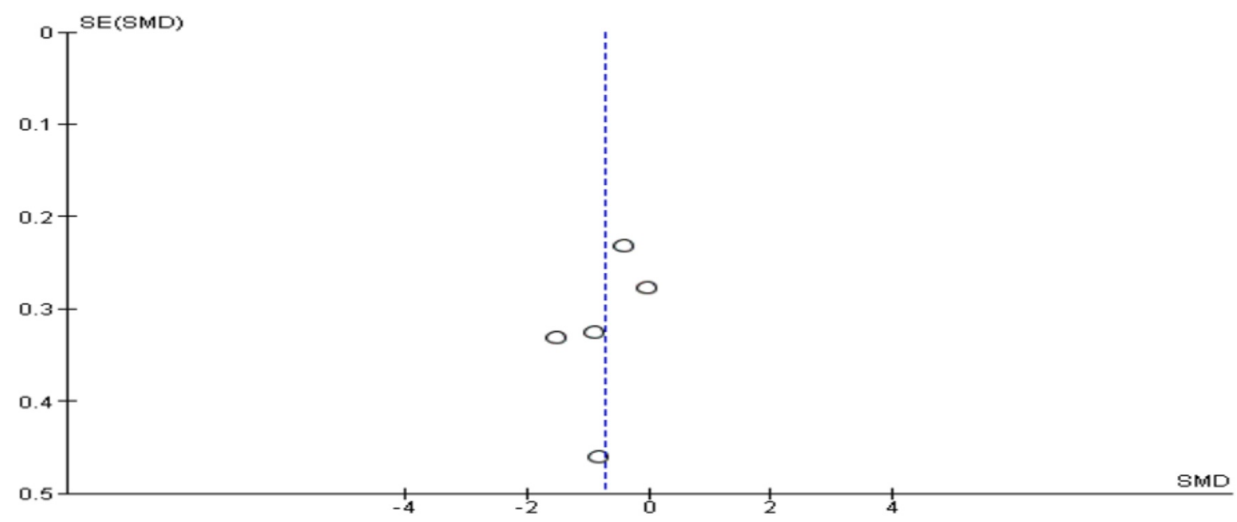

FIGURE 7: Funnel plot of meta-analysis of anxiety posttreatment.

\section{Meta-analysis of depression post-treatment}

The meta-analysis of depression symptoms was based on eight studies. The meta-analysis showed that EMDR was not better than CBT in reducing depression [SDM $(95 \% \mathrm{CI})=-0.21(-$ $0.44-0.02), p=0.08$ ]. Also, the included studies had a high incidence of heterogeneity (I2 = $70 \%$ ). These results are shown in Figure 8. Of note, there was no evidence of publication bias in the included studies in Figure 9.

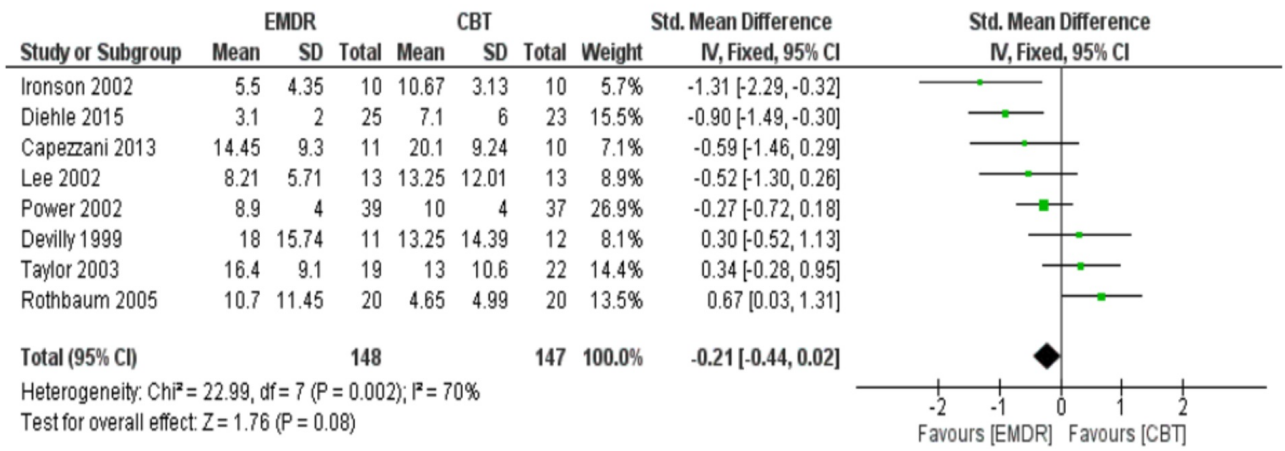

FIGURE 8: Meta-analysis of depression post-treatment.

EMDR: Eye movement desensitization and reprocessing; CBT: Cognitive behavioral therapy. 


\section{Cureus}

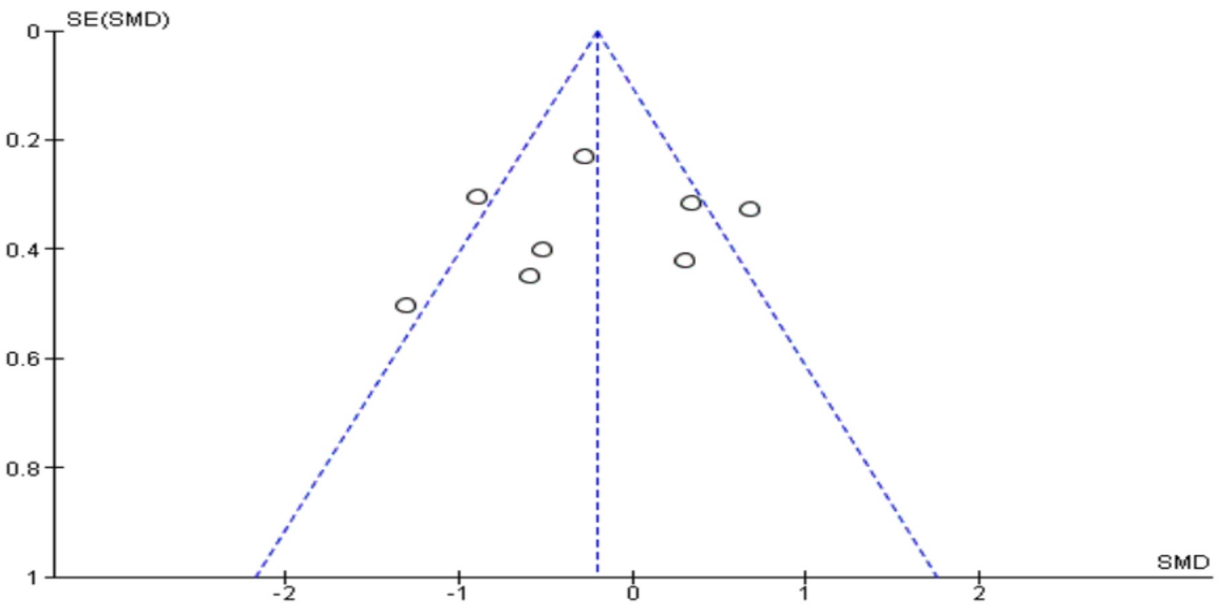

FIGURE 9: Funnel plot of meta-analysis of depression posttreatment.

\section{Meta-analysis of depression at three months follow-up}

Meta-analysis of three studies at three months follow-up showed that EMDR was not superior to CBT in reducing depression symptoms $[\mathrm{SDM}(95 \% \mathrm{CI})=0.04(-0.38-0.46), \mathrm{p}=0.86]$. Also, the included studies had a high incidence of heterogeneity ( $\mathrm{I} 2=58 \%$ ) in Figure 10 . No evidence of publication as shown in the funnel plot in Figure 11.

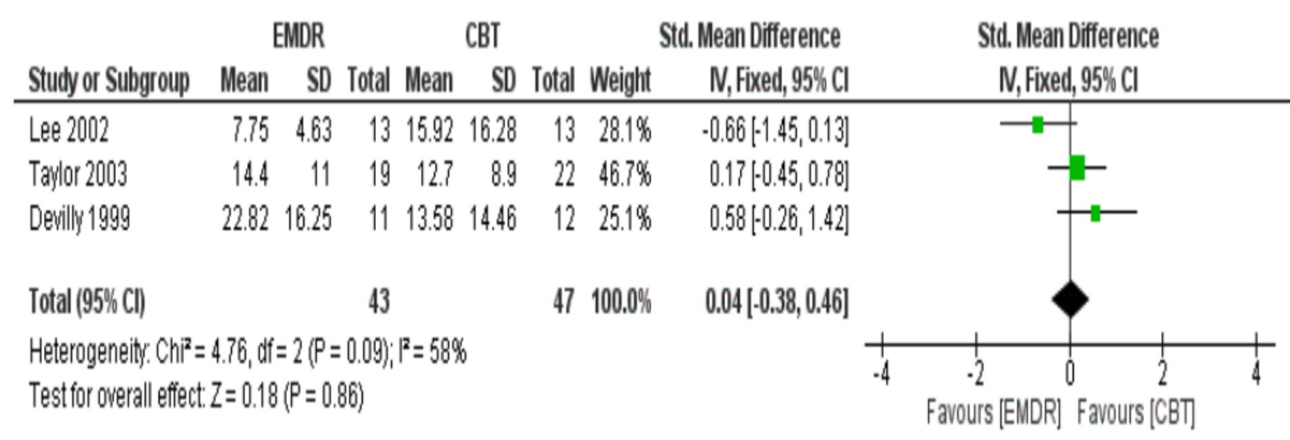

FIGURE 10: Meta-analysis of depression at three months follow-up.

EMDR: Eye movement desensitization and reprocessing; CBT: Cognitive behavioral therapy. 


\section{Cureus}

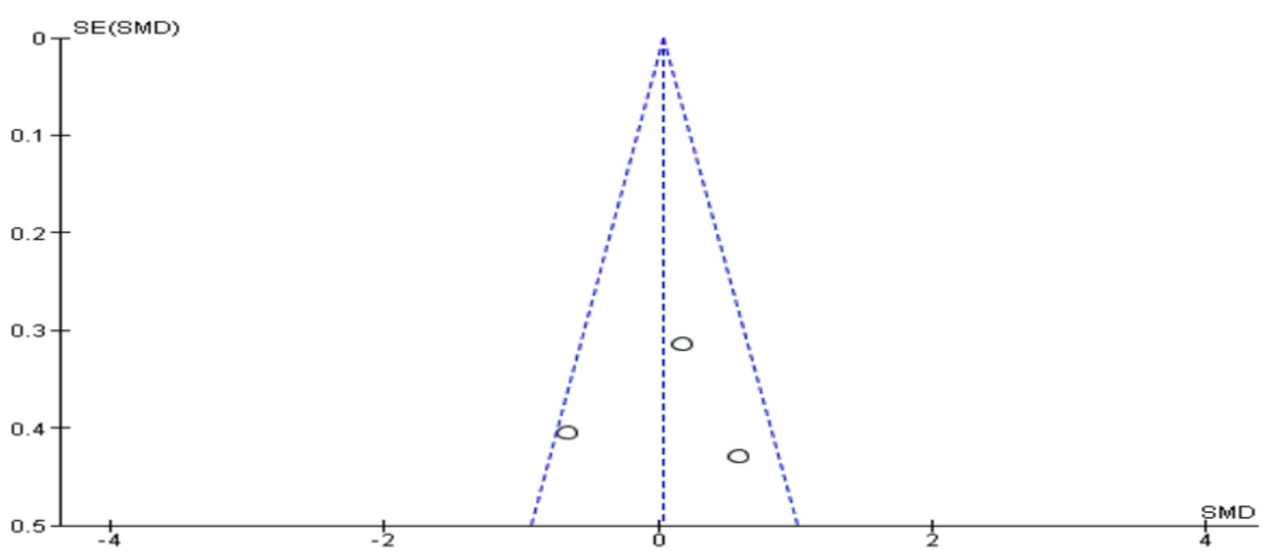

\section{FIGURE 11: Funnel plot of meta-analysis of depression at three months follow-up.}

\section{Discussion}

The objectives of this meta-analysis were to compare the efficacy of EMDR and CBT in alleviating post-traumatic symptoms, anxiety and depression. The key result of this metaanalysis is, participants treated with EMDR had better alleviation of post-traumatic symptoms as compared to participants treated with CBT $(p=0.006)$. However, the superiority of EMDR at three months follow-up was not evident. The EMDR also had a statistically significant superiority over CBT $(\mathrm{p}=0.005)$ in alleviating anxiety. Although EMDR was observed to be better than CBT in reducing depression, this difference was not statistically significant.

These findings have similarities and differences with the findings of other meta-analyses comparing EMDR and CBT. A meta-analysis by Moreno-Alcazar et al. found that EMDR was better than CBT in reducing post-traumatic symptoms among children and adolescents $(\mathrm{p}=$ 0.013) [27]. However, the difference detected by Moreno-Alcazar et al., $d=-0.49$, was higher than the difference between EMDR and CBT regarding reducing post-traumatic symptoms detected in the current meta-analysis. Similarly, a study by Chen et al. showed that EMDR was better than CBT in alleviating post-traumatic symptoms $(p=0.05)$ [28]. The difference found by Chen et al. was exactly the same as the difference determined in the current analysis, but the current analysis had stronger evidence of statistical significance. Also, the current analysis had lower heterogeneity ( $(\mathrm{I} 2=62 \%$ ) as compared to a meta-analysis by Chen et al. $(\mathrm{I} 2=70 \%)$. In the current analysis, EMDR was found to be better than CBT post-treatment; however, the study by Seidler and Wagner did not detect any superiority [11]. This difference could probably be attributed to the inclusion of more randomized trials in our recent meta-analysis compared to their study conducted in 2006. Nevertheless, at three months follow-up, the findings of the current meta-analysis were consistent with the findings of Seidler and Wagner.

The results of the meta-analysis by Lee and Ho (2012) and Davidson and Parker (2001) found inconsistent results with the current meta-analysis on the efficacy of EMDR and CBT [29, 30]. Their meta-analysis did not find a significant difference between EMDR and CBT $(\mathrm{p}=0.085)$ in alleviating post-traumatic symptoms while the current meta-analysis found a statistically significant difference. It is noteworthy mentioning that the Davidson and Parker (2001), Seidler and Wagner (2006), and Lee and Ho (2012) only included studies that have been published up to 2006. While our study aimed to investigate studies published before and after 2006, which will remain an important advantage of our meta-analysis compared to previous studies $[30,11,29]$. 
Our meta-analysis declared no difference between CBT and EMDR post-treatment in alleviating depression, which is incompatible with the findings of Seidler and Wagner (2001) who found that EMDR was better than CBT in reducing depression symptoms. Of note, the meta-analysis by Seidler and Wagner included seven studies while the current analysis meta-analyzed eight studies. Additionally, in agreement with the findings of Seidler and Wagner (2001), the metaanalysis by Ho and Lee (2012) found that the EMDR was better than TF-CBT in alleviating depression. In contrast, the concept that no difference between EMDR and CBT in alleviating depression was consistent with the findings of Moreno-Alcazar et al. (2012) who did not find a statistically significant difference.

Regarding reducing anxiety, the current meta-analysis reported that EMDR was better than CBT in alleviating anxiety, which is consistent with the findings of Moreno-Alcazar et al. (2017) who also found a statistically significant difference.

When translating the findings of this meta-analysis into clinical practice, there are several limitations that need to be considered. For instance, there are few numbers of included participants in the analyzed randomized trials. We also searched only three major databases; however, we considered the manual searching to cover more articles. The subgroup metaanalysis of post-traumatic symptoms and depression at three months follow-up, comprised a few number of analyzed studies. Our inferences must be interpreted cautiously due to the presence of bias in more than one domain of included trials. We also did not conduct metaregression of various variables such as age and sex due to a small number of included studies. Future large-scale randomized trials are warranted with longer follow-up periods and adherence to well-designed protocols.

\section{Conclusions}

The results of this meta-analysis suggested that EMDR is better than CBT in reducing posttraumatic symptoms and anxiety. However, there was no difference reported in reducing depression. Large population randomized trials with longer follow-up are recommended to build conclusive evidence.

\section{Additional Information \\ Disclosures}

Human subjects: All authors have confirmed that this study did not involve human participants or tissue. Animal subjects: All authors have confirmed that this study did not involve animal subjects or tissue. Conflicts of interest: In compliance with the ICMJE uniform disclosure form, all authors declare the following: Payment/services info: All authors have declared that no financial support was received from any organization for the submitted work. Financial relationships: All authors have declared that they have no financial relationships at present or within the previous three years with any organizations that might have an interest in the submitted work. Other relationships: All authors have declared that there are no other relationships or activities that could appear to have influenced the submitted work.

\section{References}

1. American Psychiatric Association: Diagnostic and Statistical Manual of Mental Disorders (DSM- ${ }^{\circledR}$ ). APA Publishing, Washington, DC; 2013.

2. World Health Organisation: The ICD-10 Classification of Mental and Behavioural Disorders: Diagnostic Criteria for Research. WHO, Geneva; 1993.

3. Diehle J, Opmeer BC, Boer F, Mannarino AP, Lindauer RJL: Trauma-focused cognitive behavioral therapy or eye movement desensitization and reprocessing: what works in children with posttraumatic stress symptoms? A randomized controlled trial. Eur Child Adolesc 
Psychiatry. 2015, 24:227-236. 10.1007/s00787-014-0572-5

4. Dückers ML, Alisic E, Brewin CR: A vulnerability paradox in the cross-national prevalence of post-traumatic stress disorder. Br J Psychiatry. 2016, 209:300-305. 10.1192/bjp.bp.115.176628

5. Bremner JD, Southwick SM, Johnson DR, Yehuda R, Charney DS: Childhood physical abuse and combat-related posttraumatic stress disorder in Vietnam veterans. Am J Psychiatry. 1993, 150:235-239. 10.1176/ajp.150.2.235

6. Kessler RC, Sonnega A, Bromet E, Hughes M, Nelson CB: Posttraumatic stress disorder in the National Comorbidity Survey. Arch Gen Psychiatry. 1995, 52:1048-1060.

10.1001/archpsyc.1995.03950240066012

7. Kilpatrick DG, Resnick HS, Milanak ME, Miller MW, Keyes KM, Friedman MJ: National estimates of exposure to traumatic events and PTSD prevalence using DSM-IV and DSM-5 criteria. J Trauma Stress. 2013, 26:537-547. 10.1002/jts.21848

8. Lancaster CL, Teeters JB, Gros DF, Back SE: Posttraumatic stress disorder: overview of evidence-based assessment and treatment. J Clin Med. 2016, 5:105. 10.3390/jcm5110105

9. Gillespie K, Duffy M, Hackmann A, Clark DM: Community based cognitive therapy in the treatment of post-traumatic stress disorder following the Omagh bomb. Behav Res Ther. 2002, 40:345-357. 10.1016/S0005-7967(02)00004-9

10. Kar N: Cognitive behavioral therapy for the treatment of post-traumatic stress disorder: a review. Neuropsychiatr Dis Treat. 2011, 7:167-181. 10.2147/NDT.S10389

11. Seidler GH, Wagner FE: Comparing the efficacy of EMDR and trauma-focused cognitivebehavioral therapy in the treatment of PTSD: a meta-analytic study. Psychol Med. 2006, 36:1515-1522. 10.1017/S0033291706007963

12. Rothbaum BO, Astin MC, Marsteller F: Prolonged exposure versus eye movement desensitization and reprocessing (EMDR) for PTSD rape victims. J Trauma Stress. 2005, 18:607-616. 10.1002/jts.20069

13. Arabia E, Manca ML, Solomon RM: EMDR for survivors of life-threatening cardiac events: results of a pilot study. J EMDR Pract Res. 2011, 5:2-13. 10.1891/1933-3196.5.1.2

14. Chen Y-R, Hung K-W, Tsai J-C, et al.: Efficacy of eye-movement desensitization and reprocessing for patients with posttraumatic-stress disorder: a meta-analysis of randomized controlled trials. PLOS One. 2014, 9:e103676. 10.1371/journal.pone.0103676

15. Valiente-Gómez A, Moreno-Alcázar A, Treen D, Cedrón C, Colom F, Perez V, Amann BL: EMDR beyond PTSD: a systematic literature review . Front Psychol. 2017, 8:1668. 10.3389/fpsyg.2017.01668

16. de Roos C, Greenwald R, den Hollander-Gijsman M, Noorthoorn E, van Buuren S, de Jongh A: A randomised comparison of cognitive behavioural therapy (CBT) and eye movement desensitisation and reprocessing (EMDR) in disaster-exposed children. Eur J Psychotraumatol. 2011, 2:10.3402/ejpt.v2i0.5694

17. Devilly GJ, Spence SH: The relative efficacy and treatment distress of EMDR and a cognitivebehavior trauma treatment protocol in the amelioration of posttraumatic stress disorder. J Anxiety Disord. 1999, 13:131-157. 10.1016/S0887-6185(98)00044-9

18. Ironson G, Freund B, Strauss J, Williams J: Comparison of two treatments for traumatic stress: a community-based study of EMDR and prolonged exposure. J Clin Psychol. 2002, 58:113-128. 10.1002/jclp.1132

19. Jaberghaderi N, Greenwald R, Rubin A, Zand SO, Dolatabadi S: A comparison of CBT and EMDR for sexually-abused Iranian girls. Clin Psychol Psychother. 2004, 11:358-368. 10.1002/cpp.395

20. Lee C, Gavriel H, Drummond P, Richards J, Greenwald R: Treatment of PTSD: stress inoculation training with prolonged exposure compared to EMDR. J Clin Psychol. 2002, 58:1071-1089. 10.1002/jclp.10039

21. Power K, McGoldrick T, Brown K, Buchanan R, Sharp D, Swanson V, Karatzias A: A controlled comparison of eye movement desensitization and reprocessing versus exposure plus cognitive restructuring versus waiting list in the treatment of post-traumatic stress disorder. Clin Psychol Psychother. 2002, 9:299-318. 10.1002/cpp.341

22. de Roos C, van der Oord S, Zijlstra B, Lucassen S, Perrin S, Emmelkamp P, de Jongh A: Comparison of eye movement desensitization and reprocessing therapy, cognitive behavioral writing therapy, and wait-list in pediatric posttraumatic stress disorder following singleincident trauma: a multicenter randomized clinical trial. J Child Psychol Psychiatry. 2017, 58:1219-1228. 10.1111/jcpp.12768 
23. Taylor S, Thordarson DS, Maxfield L, Fedoroff IC, Lovell K, Ogrodniczuk J: Comparative efficacy, speed, and adverse effects of three PTSD treatments: exposure therapy, EMDR, and relaxation training. J Consult Clin Psychol. 2003, 71:330-338. 10.1037/0022-006X.71.2.330

24. Nijdam MJ, Gersons BP, Reitsma JB, de Jongh A, Olff M: Brief eclectic psychotherapy v. eye movement desensitisation and reprocessing therapy for post-traumatic stress disorder: randomised controlled trial. Br J Psychiatry. 2012, 200:224-231. 10.1192/bjp.bp.111.099234

25. Capezzani L, Ostacoli L, Cavallo M, et al.: EMDR and CBT for cancer patients: comparative study of effects on PTSD, anxiety, and depression. J EMDR Prac Res. 2013, 7:134-143. 10.1891/1933-3196.7.3.134

26. van den Berg DP, de Bont PA, van der Vleugel BM, de Roos C, de Jongh A, van Minnen A, van der Gaag M: Trauma-focused treatment in PTSD patients with psychosis: symptom exacerbation, adverse events, and revictimization. Schizophr Bull. 2015, 42:693-702. 10.1093/schbul/sbv172

27. Moreno-Alcázar A, Treen D, Valiente-Gómez A, Sio-Eroles A, Pérez V, Amann BL, Radua J: Efficacy of eye movement desensitization and reprocessing in children and adolescent with post-traumatic stress disorder: a meta-analysis of randomized controlled trials. Front Psychol. 2017, 8:1750. 10.3389/fpsyg.2017.01750

28. Chen L, Zhang G, Hu M, Liang X: Eye movement desensitization and reprocessing versus cognitive-behavioral therapy for adult posttraumatic stress disorder: systematic review and meta-analysis. J Nerv Ment Dis. 2015, 203:443-451. 10.1097/NMD.0000000000000306

29. Ho MSK, Lee CW: Cognitive behaviour therapy versus eye movement desensitization and reprocessing for post-traumatic disorder - is it all in the homework then?. Eur Rev Appl Psychol. 2012, 62:253-260. 10.1016/j.erap.2012.08.001

30. Davidson PR, Parker KC: Eye movement desensitization and reprocessing (EMDR): a metaanalysis. J Consult Clin Psychol. 2001, 69:305-316. 10.1037/0022-006X.69.2.305 